\title{
Evaluación y tratamiento del niño con síntomas del tracto urinario inferior (LUTS)
}

\author{
Gustavo Adolfo Guerrero-Tinoco' ${ }^{1}$, Mónica Isabel Cano-Martínez², Juan José Vanegas-Ruiz ${ }^{3}$
}

\section{RESUMEN}

La disfunción miccional es un trastorno del llenado o vaciado de la vejiga en niños sin alteraciones neurológicas o anatómicas. Los síntomas del tracto urinario inferior (LUTS, por la sigla en inglés de Lower urinary tract symptoms) son un motivo frecuente de consulta con el pediatra, el nefrólogo o el urólogo pediatra, e incluso con el neurólogo y el psicólogo infantil. Se le considera una enfermedad relativamente benigna lo que en ocasiones genera desinterés entre médicos y familiares y lleva a consultas tardías e interpretación inadecuada de los síntomas. Manifestaciones como urgencia, incontinencia, enuresis, goteo posmiccional, infecciones urinarias, vulvovaginitis recurrente y constipación, en un niño sin enfermedad neurológica, deben hacer pensar al médico en disfunción miccional para enfocar oportunamente el diagnóstico, restablecer la calidad de vida del paciente, evitar las infecciones urinarias y el daño renal subsecuente e irreversible secundario a un diagnóstico tardío. Las recomendaciones actuales hacen énfasis en un enfoque menos invasivo, tratamiento conservador, manejo de la constipación y reentrenamiento vesical. Este artículo discute la evaluación, el diagnóstico y el tratamiento correctos de niños con LUTS.

\section{PALABRAS CLAVE}

Incontinencia; Micción Disfuncional; Niño; Tracto Urinario Inferior; Vejiga

1 Nefrólogo pediatra, Hospital Infantil Napoleón Franco Pareja, Cartagena de Indias, Colombia.

2 Pediatra, Universidad de Antioquia, Medellín, Colombia.

3 Nefrólogo pediatra, Hospital Pablo Tobón Uribe, Medellín, Colombia.

Correspondencia: Gustavo Adolfo Guerrero Tinoco; gusgueti@hotmail.com

Recibido: septiembre 12 de 2016

Aceptado: febrero 28 de 2017

Cómo citar: Guerrero-Tinoco GA, Cano-Martínez MI, Vanegas-Ruiz JJ. Evaluación y tratamiento del niño con síntomas del tracto urinario inferior (LUTS). latreia. 2018 EneMar;31(1):51-64. D0I 10.17533/udea.iatreia.v31n1a05. 


\section{SUMMARY}

\section{Evaluation and management of children with lower urinary tract symptoms (LUTS)}

Voiding dysfunction is a disorder of the bladder filling or emptying in children without neurological or anatomical disorders. Lower urinary tract symptoms (LUTS) are a frequent reason for consulting the pediatrician, nephrologist or pediatric urologist, and even the neurologist and child psychologist. It is considered a relatively benign disease that sometimes generates disinterest among doctors and families, leading to late consultation and inadequate interpretation of symptoms. Urgency, incontinence, enuresis, post-roid dribbling, urinary tract infections, recurrent vulvovaginitis and constipation in children without neurological disease should lead to consider the possibility of voiding dysfunction, in order to recognize it timely, restore the quality of life, prevent urinary tract infection and the irreversible kidney damage secondary to delayed diagnosis. Current recommendations emphasize on a less invasive approach, conservative treatment, management of constipation and bladder retraining. This article discusses the correct assessment, diagnosis and management of children with LUTS.

\section{KEY WORDS}

Bladder; Child; Incontinence; Lower Urinary Tract; Voiding Dysfuncional

\section{INTRODUCCIÓN}

Los síntomas del tracto urinario inferior (LUTS, por la sigla en inglés de lower urinary tract symptoms) en la infancia son un problema común en la consulta. Los niños con disfunción del tracto urinario inferior (LUTD, por la sigla en inglés de lower urinary tract dysfunction) pueden presentarse con incontinencia, infección del tracto urinario, reflujo vesicoureteral (RVU) y estreñimiento solos o en combinación (1). Se calcula que alrededor de $20 \%$ a $30 \%$ de los niños experimentan síntomas relacionados con disfunción miccional. Diversos estudios han encontrado tasas de incontinencia y enuresis hasta del $20 \%$ en esta población $(1,2)$. En la edad escolar estos síntomas por sí solos representan de 30 \% a 40 \% de las consultas al nefrólogo pediatra $(1,3)$.

\section{FISIOPATOLOGÍA}

El tracto urinario funciona bien si es capaz de almacenar orina a bajas presiones y sin escape y de vaciarse totalmente sin dejar residuo (4). Las dos funciones principales del tracto urinario inferior son el almacenamiento de la orina a baja presión (fase de llenado) y su expulsión en un tiempo y lugar apropiados (fase miccional) (4).

El almacenamiento vesical depende del volumen o capacidad vesical (CV en $\mathrm{mL}$ ) y de la capacidad del esfínter interno (cuello de la vejiga y uretra proximal) de permanecer cerrado durante el Ilenado (3). EI vaciamiento vesical depende de la capacidad del detrusor para contraerse y generar presión facilitando el flujo y de la capacidad del esfínter externo para relajarse de manera coordinada y consciente (4).

El control de la fisiología vesical está dado por inervación parasimpática y simpática en la vejiga y la uretra (4). Los nervios eferentes parasimpáticos salen de la región sacra (S2-S4) y viajan a lo largo del nervio pélvico. El estímulo de los receptores parasimpáticos produce contracción del detrusor y relajación del tracto de salida de la vejiga (5). Los nervios eferentes simpáticos salen del seogmento toracolumbar de la médula espinal (T10-L2) y viajan en el nervio hipogástrico (5). Los receptores alfa-adrenérgicos se ubican en el cuello vesical y la uretra proximal, $y$ al estimularse producen contracción del esfínter interno $(3,5)$. Los receptores beta-adrenérogicos están localizados en el cuerpo vesical 8 al ser estimulados producen relajación del detrusor y permiten el llenado $(4,5)$. Además, existe una inervación somática que sale de la región sacra de la médula espinal (S2-S4), cuyas fibras viajan a lo largo del nervio pudendo y su función es regular la contracción voluntaria del esfínter urinario externo $(5,6)$.

\section{TERMINOLOGÍA}

Recientemente la ICCS (International Children's Continence Society) actualizó la terminología sobre Ia función $y$ disfunción del tracto urinario inferior, dando uniformidad y claridad a las definiciones y la 
caracterización de la disfunción miccional para diferentes disciplinas de la salud y haciendo énfasis en reconocer la estrecha relación entre las funciones del intestino y la vejiga en niños y adolescentes $(7,8)$.

\section{SÍNTOMAS DEL TRACTO URINARIO INFERIOR (LUTS)}

La terminología para LUTS se utiliza en los niños a partir de los 5 años cumplidos, edad utilizada en la quinta edición deI DSM-5 (Diagnostic and Statistical Manual of Mental Disorders) y en la CIE-10 (Clasificación Internacional de Enfermedades, décima edición) para caracterizar los trastornos de incontinencia urinaria. Para la disfunción intestinal la edad mínima es de 4 años. Sin embargo, existe variabilidad en el aspecto madurativo de la función vesical $y$ hay niños que logran el control voluntario sobre el tracto urinario inferior antes de los 5 años; por tanto, el control vesical y gastrointestinal pueden ocurrir antes, $y$ la terminología puede ser aplicable selectivamente a cohortes de niños menores (7).

La ICCS $(7,8)$ define y aogrupa los términos de la siguiente manera:

\section{Síntomas en la fase de almacenamiento}

Aumento o disminución de la frecuencia de la micción: la frecuencia miccional es variable y está influenciada por la edad, la ingesta de líquidos y la diuresis, más que por la capacidad de la vejiga. Se considera como aumento en la frecuencia en los niños cuando hay ocho o más micciones durante el día, $y$ frecuencia urinaria disminuida cuando hay tres o menos por día.

Incontinencia: es la pérdida involuntaria de la orina. Puede ser continua o intermitente; la primera consiste en la pérdida constante (día y noche), por lo general asociada con malformaciones congénitas (uréter ectópico, extrofia vesical), pérdida de la función del esfínter uretral externo (esfinterotomía externa) o causas iatrogénicas (fístula vesicovaginal). La incontinencia intermitente es la pérdida de orina diurna o nocturna en cantidades pequeñas.

La incontinencia intermitente que se produce mientras se está despierto se denomina incontinencia diurna y la que se produce exclusivamente en la noche o durante los períodos de sueño, se denomina enuresis, que se clasifica así: 1) enuresis monosintomática: cuando no hay ningún otro LUTS ni antecedentes de disfunción vesical; 2) enuresis no monosintomática: la que se acompaña de otros síntomas.

Urgencia: se refiere a la sensación repentina e inesperada de una necesidad inmediata de orinar. Puede ser un siogno de hiperactividad de la vejiga.

Nicturia: es la queja de que el niño debe despertar en la noche para orinar. La definición es relevante desde los 5 años, es común en escolares y no necesariamente indica LUTD.

\section{Síntomas en la fase de eliminación}

Vacilación: dificultad para iniciar la micción cuando el niño está listo para eliminar.

Esfuerzo: el niño se queja de tener que hacer un esfuerzo intenso para aumentar la presión intrabdominal (por ejemplo, la maniobra de Valsalva) con el fin de iniciar y mantener la micción. Es relevante en todos los grupos de edad.

Chorro débil: expulsión observada del flujo de orina con fuerza débil.

Intermitencia: la micción no se produce en un flujo continuo, sino que tiene varias paradas breves con inicio a borbotones (en varios chorros). Se considera que es fisiológica hasta los 3 años si no va acompañada por el esfuerzo.

Disuria: ardor o molestia durante la micción. Si es al inicio de esta sugiere una fuente uretral del dolor, mientras que la disuria al terminar la micción sugiere un origen en la vejiga.

\section{Otros síntomas}

Maniobras de retención: estrategias retentivas observables utilizadas para posponer la micción o suprimir la sensación de urgencia que pueden estar asociadas con hiperactividad de la vejiga. El niño puede o no ser plenamente consciente de los efectos de estas maniobras, pero por lo general son obvias para los cuidadores. Comportamientos comunes incluyen pararse en las puntas de los pies, cruzar con fuerza las piernas, agarrar o hacer presión en los genitales, en el abdomen o en el periné (por ejemplo, posición en cuclillas con el talón presionando el periné o sentarse en el borde de una silla). 
Sensación de vaciado incompleto: puede manifestarse como la necesidad de volver al baño a orinar de nuevo o el aumento en la frecuencia miccional.

Retención urinaria: se refiere a la sensación de incapacidad para orinar a pesar de un esfuerzo persistente teniendo la vejiga completamente distendida.

Goteo posmiccionaI: pérdida involuntaria de orina inmediatamente después de terminar la micción. Este síntoma puede estar asociado con reflujo vaginal en las niñas o siringocele en los niños (dilatación quística de la glándula de Cowper).

Dispersión del flujo urinario: la micción es dispersa en forma de aerosol en lugar de ser un chorro único. Se relaciona con una obstrucción mecánica en el meato, por ejemplo, estenosis.

Dolor genital, vesical y en el tracto urinario inferior: en las niñas son comunes el dolor vaginal, el dolor o la presión suprapúbica y el prurito vulvar con irritación local secundaria a la incontinencia. En hombres jóvenes se pueden hallar dolor en el pene y priapismo episódico, asociados con la vejiga llena, el estreñimiento o la retención de la orina dentro del prepucio fimótico.

\section{DIAGNÓSTICO}

La historia clínica y el examen físico completos son las principales herramientas para la evaluación de los niños y adolescentes con LUTS y pueden llevar al diagnóstico sin necesidad de exámenes adicionales (9). En el examen físico se deben buscar las malformaciones congénitas y las manifestaciones cutáneas de espina bífida mediante la inspección de las regiones genital y sacra; explorar para siognos de déficits neurológicos, los reflejos de las extremidades, la sensación del área perineal, el tono anal y el reflejo bulbocavernoso (10). En la consulta inicial es de utilidad solicitar exámenes de laboratorio como uroanálisis, urocultivo, ecografía renal y de las vías urinarias con medición de los volúmenes pre- $y$ posmiccional. Los estudios urodinámicos específicos que ayudan a la evaluación se clasifican en invasivos y no invasivos $(7,8)$.

Diario miccional: es el registro de la ingesta de líquidos y el volumen de orina en cada micción durante días o semanas (tanto diurnos y nocturnos), anotados en la fecha $y$ horario correspondientes. El diario miccional completo incluye los episodios de incontinencia con los volúmenes urinarios de 7 noches para evaluar la enuresis, $y$ un registro del volumen y la frecuencia diurnos de 48 horas para evaluar los $\operatorname{LUTD}(7,8)$. Además de los episodios de incontinencia y enuresis, también incluye síntomas relevantes como urgencia, dolor, escape, entre otros (11).

UItrasonido: es un estudio muy útil en la evaluación del tracto urinario inferior. Permite cuantificar el residuo posmiccional (RPM), que se considera elevado en los siguientes casos:

Niños de 4 a 6 años: RPM único mayor de $30 \mathrm{~mL} o$ del $21 \%$ de la capacidad vesical (CV); la CV se determina por el volumen miccional ( $\nabla M)$ más el residuo posmiccional (RPM) y se expresa como el porcentaje de la capacidad vesical estimada (CVE), cuya fórmula fue validada (CVE = [edad (años) + 1] x 30) (12). Un RPM repetidamente mayor de $20 \mathrm{~mL}$ o del $10 \%$ de la CVE se considera muy significativo $(7,8)$.

Niños de 7 a 12 años: RPM único mayor de $20 \mathrm{~mL}$ o del $15 \%$ de la $C V$ o repetidamente mayor de $10 \mathrm{~mL}$ o del $6 \%$ de la $C V$ se considera elevado $(7,8)$.

Cistouretrografía miccional (CUM): está indicada en pacientes con infecciones documentadas del tracto urinario (ITU) (3).

Uroflujometría: es un procedimiento no invasivo de medición del flujo urinario, que se define como el volumen de orina que pasa por la uretra en la unidad de tiempo, expresado en $\mathrm{mL} / \mathrm{seg}$. Evalúa el flujo máximo (Qmáx), el flujo promedio (Qprom), el volumen total evacuado y el tiempo total de la micción. Permite observar la morfología de la curva obtenida durante la micción (Figura 1). Constituye uno de los estudios iniciales para evaluar el vaciado vesical. La uroflujometría se puede hacer con o sin electromiografía (EMG) de los músculos perineales. La uroflujometría normal refleja la cinética de la contracción del músculo detrusor frente a la resistencia que genera la uretra (13).

Urodinamia: evalúa los parámetros de llenado y vaciamiento vesicales. Es un procedimiento invasivo en el que la vejiga se llena con agua (preferiblemente) o $\mathrm{CO}_{2}$, mientras se controlan la presión intravesical mediante un catéter en la vejiga y la intrabdominal con un catéter rectal. Las fases de la urodinamia incluyen 
la uroflujometría, la cistometría y el estudio presiónvolumen. En la uroflujometría libre se miden, como ya se dijo, el Qmax, el Qprom, el volumen total evacuado y el tiempo total de la micción. En la cistometría se monitorizan los cambios de presión vesical durante la fase de llenado y se evalúan la sensibilidad, la capacidad, la acomodación vesical, la actividad del detrusor y la función uretral durante dicha fase. En el estudio presión-volumen se evalúan la actividad del detrusor y la función uretral durante la fase de vaciamiento (13, 14). No todo paciente con LUTS requiere un estudio urodinámico completo. Las indicaciones para la urodinamia son las siguientes $(3,7,8)$ : disfunción vesical neurogénica, examen neurológico anormal, micción disfuncional severa, falla del tratamiento médico, presentación clínica anormal, evaluación preoperatoria. En niños se prefieren los procedimientos no invasivos como la uroflujometría no invasiva, la electromiografía perineal y la cuantificación del RPM (3).

Videourodinamia: es la visualización fluoroscópica del tracto urinario inferior mediante la cistouretrografía que se hace simultáneamente con la cistometría y

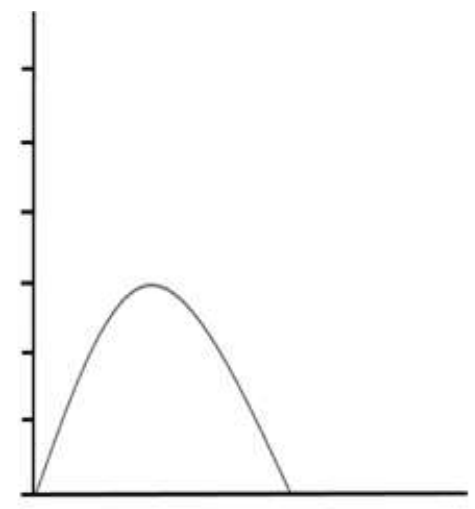

Figura 1. Curva en campana: paciente sano

con los estudios de presión y flujo. Consiste en la instilación de un medio de contraste y en captar mediante fluoroscopia las imágenes del tracto urinario (15). Es un estudio invasivo indicado en casos seleccionados: paciente con enfermedad neurológica que afecte al tracto urinario inferior, incontinencia urinaria masculina, incontinencia urinaria femenina recidivante $y$ compleja, obstrucción del tracto urinario femenino, paciente joven con antecedente de obstrucción del tracto urinario de salida o con antecedente de cirugía previa y paciente pediátrico con micción disfuncional o enfermedad neurológica (13).

\section{SÍNDROMES CLÍNICOS RELACIONADOS CON LA DISFUNCIÓN MICCIONAL}

Utilizando las definiciones del CIE-10 y los criterios del DSM-5, para el diagnóstico de incontinencia se requieren una edad mínima de 5 años, más de un episodio por mes y una duración de al menos 3 meses (16).

Enuresis: es un síntoma de la incontinencia intermitente que se produce durante los períodos de sueño. Se considera importante cuando su frecuencia es mayor de 4 veces por semana e infrecuente cuando ocurre menos de 4 veces por semana $(7,8)$. Se subdivide, según su aparición, en secundaria en los niños que han tenido un período seco previo de más de 6 meses, y primaria en el caso contrario. La enuresis secundaria tiene mayor asociación con trastornos del comportamiento que requieren investigación $(7,8)$. Se reconocen tres principales formas fisiopatológicas de la enuresis: 1) la poliuria nocturna, en la que hay disminución de la vasopresina nocturna con producción de orina por encima del $130 \%$ de la capacidad vesical (CVE); 2) se relaciona con disminución del almacenamiento de la vejiga durante la noche; 3 ) se refiere a la incapacidad para despertar 
en respuesta a los impulsos originados por la distensión de la vejiga (17).

Disfunción de la vejiga y el intestino (DVI): los tractos genitourinario (TGU) y gastrointestinal (TGI) comparten el origen embriológico (endodermo), la inervación (parasimpático S2, S4, simpático L1, L3), el espacio anatómico y la relación con la musculatura del piso pélvico (18). Debido a esta relación entre la vejiga y el intestino, los trastornos concomitantes del vaciado intestinal y vesical se han etiquetado como DVI. Este es un término general que se subdivide en disfunción del tracto urinario inferior (LUTD) en niños mayores de 5 años y disfunción intestinal en mayores de 4 años, en niños que no tienen ninguna anormalidad neurológica identificada (19).

Vejiga hiperactiva: se caracteriza por una falta de inhibición central de la contracción del detrusor (hiperactividad del detrusor en la urodinamia), aumento en la frecuencia miccional, urgencia urinaria y nicturia, con o sin incontinencia urinaria, en ausencia de infección del tracto urinario o de otra enfermedad obvia. Ha reemplazado el término "vejiga inestable de la niñez" (20). En la uroflujometría se encuentra un flujo miccional en forma de torre, que se caracteriza por una curva alta y de duración corta (Figura 2).

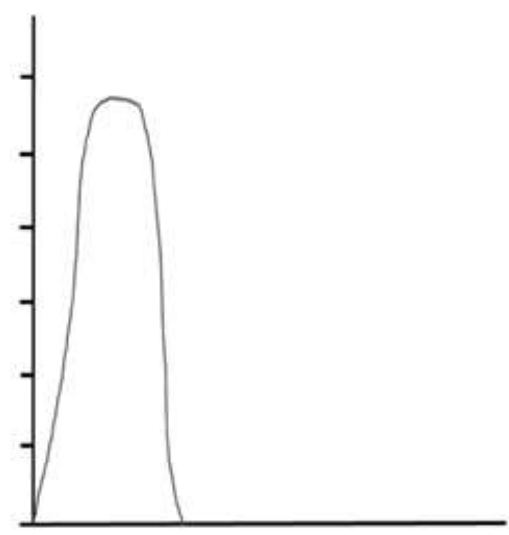

Figura 2. Curva en forma de torre: micción explosiva relacionada con una contracción no inhibida

Aplazamiento miccional: se presenta en los niños que habitualmente retrasan de manera voluntaria la micción mediante maniobras retentivas. Se observa con frecuencia cuando el niño está haciendo actividades diurnas como jugar con sus coetáneos $(7,8)$. Este comportamiento se asocia a menudo con una baja frecuencia miccional y sensación de urgencia cuando la vejiga está sobredistendida. El niño pospone el vaciado de la vejiga el mayor tiempo posible $y$ en consecuencia moja su ropa interior; este aplazamiento se puede asociar con el estreñimiento, porque el niño también puede evitar una defecación potencialmente dolorosa. Con el tiempo, el estreñimiento puede predisponer a incontinencia fecal. Estos niños sufren a menudo comorbilidades psicológicas o del comportamiento como el trastorno oposicionista desafiante (21).

Vejiga hipoactiva: antes se llamaba "vejiga perezosa”. Este término clínico está reservado para los niños que necesitan aumentar la presión intrabdominal para iniciar, mantener o completar la micción. Pueden tener baja frecuencia miccional a pesar de hidratación adecuada, pero también pueden presentar aumento de la frecuencia debido al vaciado incompleto. En la urodinamia invasiva se observa un patrón de flujo urinario interrumpido e hipoactividad del detrusor (Figura 3). Estos niños presentan frecuencia urinaria disminuida, orinan 2 a 4 veces al día, no orinan al levantarse y rara vez orinan en el colegio $(7,8)$. 


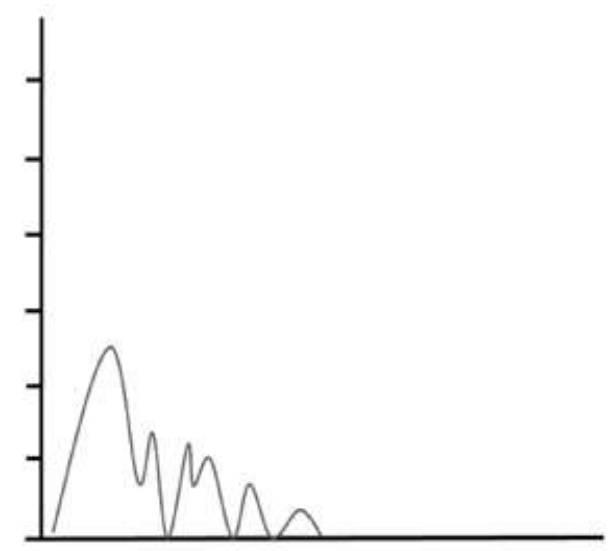

Figura 3. Curva en forma intermitente: picos pequeños con segmentos donde el flujo alcanza el cero (cesa completamente el flujo urinario)

Incoordinación detrusor esfínter: micción disfuncional: el niño con disfunción miccional contrae habitualmente el esfínter uretral externo o el suelo pélvico durante la micción, impidiendo el vaciado vesical.
En la urodinamia se evidencia un patrón en stacatto (vaciamiento en oleadas) o de micción fragmentada (Figura 4). Este término se usa en pacientes neurológicamente sanos $(7,8)$.

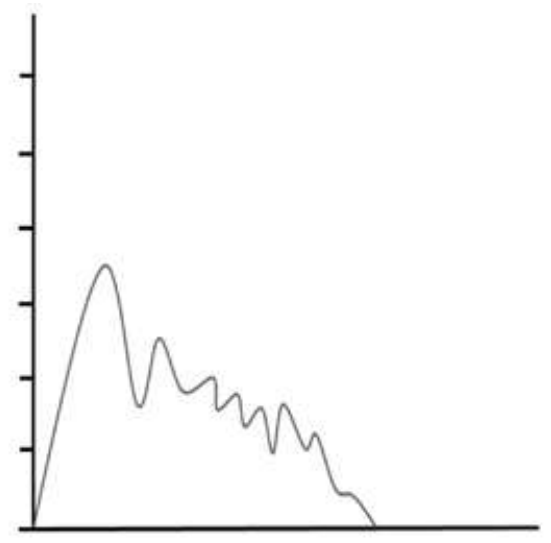

Figura 4. Curva en staccato: flujo irregular, fluctuante y continuo (nunca alcanza el 0), por incoordinación de la vejiga y el piso pélvico

Obstrucción de la salida de la vejiga: se refiere a un impedimento del flujo de orina durante la micción. Puede ser mecánico o funcional, estático o dinámico, y se caracteriza por el aumento de la presión del detrusor y una tasa de flujo urinario reducido durante los estudios de presión-flujo $(7,8)$. En la urodinamia se observa la curva en forma de meseta (plateau) (Figura 5).
Incontinencia de esfuerzo: pérdida involuntaria de orina con un esfuerzo físico que aumenta la presión intrabdominal, por ejemplo al toser o estornudar $(7,8)$.

Reflujo vaginal: se presenta en niñas prepúberes que experimentan incontinencia diurna en cantidades moderadas, poco después de la micción normal. Es consecuencia de la micción con las piernas aducidas, 


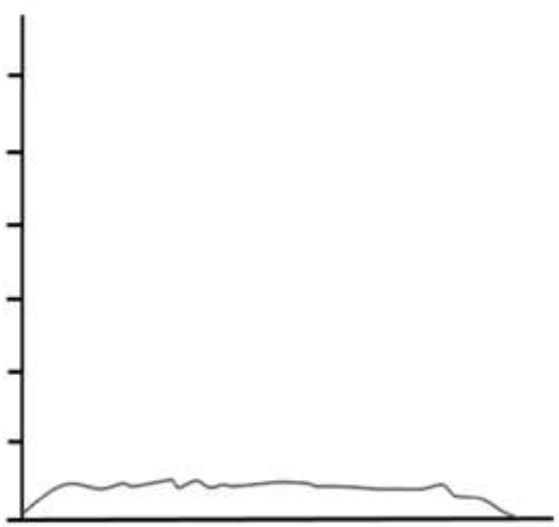

Figura 5. Curva en forma de meseta (plateau): flujo prolongado y de baja amplitud (aplanada) sugestiva de obstrucción del tracto urinario inferior

lo que lleva al atrapamiento de orina en el introito vaginal. Puede estar asociado con adherencias labiales por inflamación localizada. No se asocia con otros síntomas del tracto urinario inferior $(7,8)$.

Incontinencia durante la risa: síndrome raro en el que se produce la micción completa específicamente durante la risa o inmediatamente después de reír. La función vesical es normal cuando el niño no se está riendo. Se debe diferenciar de la vejiga hiperactiva, el aplazamiento de la micción o la vejiga hipoactiva. Se han obtenido buenos resultados con la uroterapia y la reeducación vesical (18).

Frecuencia urinaria diurna aumentada: se presenta cuando el niño tiene necesidad frecuente de orinar, asociada con pequeños volúmenes de micción, y sucede únicamente durante el día. La frecuencia miccional es al menos una vez por hora, con un volumen promedio de eliminación disminuido. La incontinencia es rara y no hay nicturia. Deben ser excluidas comorbilidades como polidipsia, diabetes mellitus, diabetes insípida nefrogénica e infección urinaria $(7,8)$.

Disfunción cuello-vejiga: se refiere a un retardo en la apertura del cuello de la vejiga, que lleva a un flujo reducido a pesar de una contracción adecuada o elevada del detrusor. Se puede diagnosticar con una uroflujometría con EMG, observando un retraso en el intervalo entre el inicio de la relajación del suelo pélvico y el comienzo de la micción (22).
Constipación funcional: la Sociedad Norteamericana para la Gastroenterología y la Nutrición Pediátricas (NASPGN, por su sigla en inglés) la define como "un retraso o dificultad en la defecación, presente por 2 semanas o más, $\searrow$ suficiente para causarle molestia al paciente" (23).

\section{TRATAMIENTO}

Está encaminado a reducir los síntomas y restaurar la función del tracto urinario inferior $(24,25)$. La ICCS ha publicado varios documentos en los que describen guías de tratamientos para LUTS y sus comorbilidades (24-29). En la Figura 6 se presenta una estrategia de diagnóstico y tratamiento del niño con LUTS.

\section{Tratamiento no farmacológico}

Uroterapia: es la principal estrategia para el tratamiento de los niños con LuTS (25); se basa en el tratamiento conservador (no farmacológico) que busca rehabilitar la función del tracto urinario inferior. Se puede dividir en terapias estándar e intervenciones específicas $(7,8)$.

Según la ICCS, los componentes estándar incluyen las siguientes medidas $(7,8)$ :

- Información y desmitificación: explicación acerca de la función normal del tracto urinario inferior $y$ de cómo el niño en particular se desvía de lo normal. 


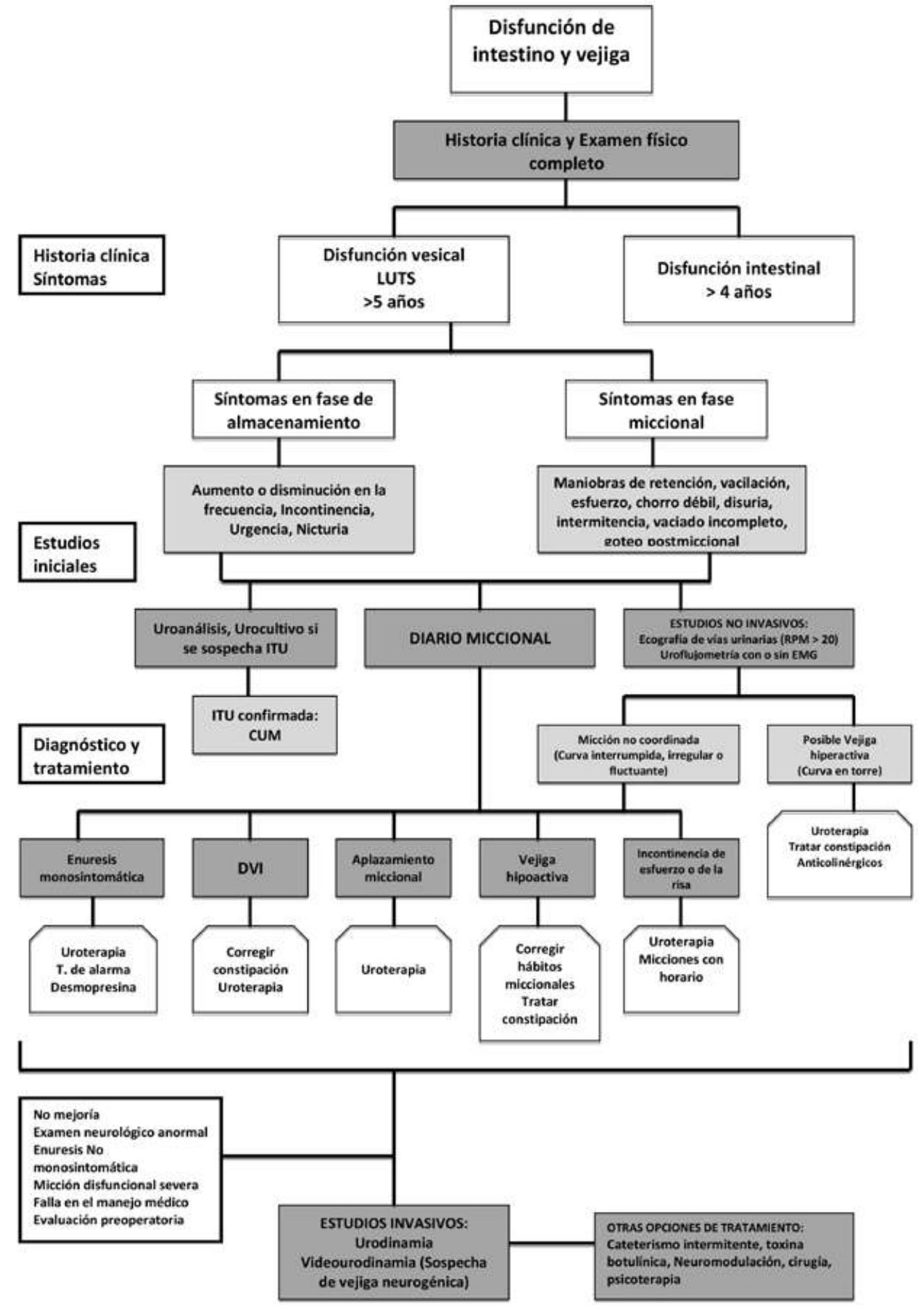

Figura 6. Algoritmo de evaluación y tratamiento del niño con síntomas del tracto urinario inferior (LUTS). CUM: cistouretrografía miccional. ITU: infección del tracto urinario. EMG: electromiografía. RPM: residuo posmiccional. DVI: disfunción vejiga intestino. 
- Instrucciones de cómo resolver la disfunción miccional con modificación del comportamiento, hábitos regulares de micción, postura adecuada al orinar, evitar maniobras de retención, no posponer la micción, orinar al levantarse y antes de acostarse, cambiar la ropa húmeda y tener hábitos intestinales regulares.

- Consejos de estilo de vida: abarcan la ingesta de líquidos y la dieta equilibrada, disminuir el consumo de cafeína, crear patrones regulares de vaciado de la vejiga y el intestino.

- Registrar los síntomas y hábitos urinarios mediante diarios miccionales.

- Dar apoyo y aliento por medio de un seguimiento regular con el cuidador.

\section{Las intervenciones específicas incluyen varias es- trategias $(7,8)$ :}

- Reentrenamiento muscular del piso pélvico (RMPP, biofeedback): sensibilización del piso pélvico para cambiar los hábitos miccionales del paciente, hacer consciente un proceso fisiológico inconsciente convirtiéndolo en un estímulo visual, auditivo o táctil.

- Ejercicios para entrenamiento del piso pélvico.

- Neuromodulación: pretende inhibir la conducta inestable de los reflejos neuronales vesicales mediante estimulación directa o acupuntura. Se basa en que las estructuras del sistema nervioso central se pueden activar por medio de corriente eléctrica y de esta forma modular la inervación de la vejiga, por lo que puede ser un tratamiento alternativo a la farmacoterapia en casos de vejiga hiperactiva (30-32).

- Estimulación con corriente eléctrica de las vísceras pelvianas o de sus nervios aferentes, por vía perineal o transcutánea.

- Cateterización intermitente.

- Terapia cognitivo-conductual y psicoterapia.

- Tratamiento de la constipación.

Terapia de alarma: se basa en el uso de un dispositivo electrónico que proporciona una fuerte señal sensorial, por lo general acústica, inmediatamente después de un episodio de incontinencia. También están disponibles alarmas con vibraciones y efectos de luz (25). Se puede utilizar durante el día o en la noche, pero su uso es más común en niños con enuresis. Es curativa en $60 \%$ de los niños mediante los efectos de acondicionamiento, aumentando el $\nabla 0-$ lumen de la vejiga (33). La duración mínima de la terapia es de 3 meses y se puede suspender después de que el niño ha logrado al menos 14 noches secas consecutivas (25). Puede haber recurrencia de la incontinencia, pero la reintroducción de esta terapia es a menudo exitosa $(9,26)$.

\section{Tratamiento farmacológico}

\section{Anticolinérgicos}

Son agentes antimuscarínicos que bloquean los receptores de acetilcolina inhibiendo las contracciones del detrusor y facilitando el llenado de la vejiga (25). Están indicados como auxiliares de la terapia con biofeedback, en pacientes con vejiga hiperactiva o capacidad vesical pequeña, cuando las intervenciones anteriores no han sido suficientes para lograr la continencia. La mayoría de los fármacos disponibles son no selectivos e interfieren con receptores muscarínicos localizados fuera de la vejiga, lo que da lugar a efectos secundarios indeseados, más comúnmente boca seca, estreñimiento y visión borrosa (34). Además, pueden producir cambios psicológicos o de la personalidad, así como activación del sistema simpático, con enrojecimiento de la cara, golpe de calor, taquicardia, somnolencia o cefalea (25). Aproximadamente $10 \%$ de los pacientes interrumpen el tratamiento debido a estos efectos secundarios (35). Los anticolinérgicos disponibles en nuestro medio son los siguientes:

Oxibutinina: es el medicamento antimuscarínico más usado en nuestro medio. Se recomienda una dosis máxima de 0,4 mg/kg por 6-12 meses con desmonte lento; hay respuesta en $61 \%$ a $86 \%$ de los casos. Se han reportado efectos adversos en $12,5 \%$ a $68 \%$ de los pacientes y debe evitarse su uso en pacientes con constipación sin tratamiento (25). Se dispone de varias presentaciones de esta droga: liberación inmediata, liberación extendida, transdérmica $(25,36)$. Es un agente antimuscarínico no selectivo por lo que su uso desencadena varios efectos adversos, más frecuentemente en niños que en adultos (boca seca, estreñimiento, visión borrosa). Las formas de liberación prolongada tienen menos efectos adversos $(37,38)$. 
Tolterodina: agente selectivo utilizado para el tratamiento de pacientes con vejiga hiperactiva; los resultados son similares a los de la oxibutinina, pero con mejor tolerancia (39). En niños también se ha documentado la superioridad de la tolterodina sobre la oxibutinina en términos de efectos adversos (40). Se recomienda una dosis de $1 \mathrm{mg}$, dos veces al día en pacientes de 5 a 10 años (41).

Solifenacina: es un nuevo agente selectivo antimuscarínico aún no aprobado en niños, aunque los estudios han probado su utilidad en niños resistentes a oxibutinina y tolterodina $(42,43)$.

Desmopresina: es un análogo de la vasopresina $u$ hormona antidiurética que hace disminuir la producción de orina durante la noche. Está indicada en pacientes con enuresis secundaria a poliuria nocturna sin hiperactividad vesical; $30 \%$ de los niños responden completamente a la desmopresina y 40 \% responden de forma parcial, pero se observa una tasa alta de recurrencia después de la interrupción de esta terapia (26). Está disponible en tabletas de 0,2 y 0,6 mg, en liofilizado oral de liberación rápida (120-360 $\mu$ g); la presentación nasal ha sido retirada en muchos países debido al aumento del riesgo de hiponatremia (44). Estudios farmacodinámicos muestran que la formulación liofilizada de $120 \mu$ g es más efectiva que su equivalente, la tableta 0,2 mg (45). Según algunos estudios $(46,47)$, la suspensión gradual de la desmopresina disminuye el riesgo de recaídas comparada con la suspensión brusca. Este es un medicamento seguro, incluso con el uso prolongado, pero se debe advertir a los cuidadores del niño de que la ingestión excesiva de líquido justo antes o después de su administración puede causar intoxicación hídrica, hiponatremia $y$ convulsiones, por lo que se debe vigilar para la aparición de náuseas, vómitos y cefalea (48).

Imipramina: es un antidepresivo tricíclico, indicado para el tratamiento de la enuresis nocturna. Su mecanismo de acción todavía no está claro, pero se ha propuesto que tiene propiedades antidiuréticas y también reduce el umbral de excitación (49). Tiene el potencial de producir efectos cardiotóxicos, por lo que se debe descartar el síndrome de QT largo antes de iniciar el tratamiento. La dosis inicial en niños es de 25-50 mg al acostarse. Se debe utilizar con precaución y como opción de tercera línea en pacientes con enuresis resistente (25).
Bloqueadores alfa-adrenérgicos: producen relajación del músculo liso y disminución en la resistencia a la salida de la orina de la vejiga. Sus efectos secundarios más comunes son hipotensión y mareos. La tamsulosina es un alfa-boqueador selectivo usado rutinariamente para facilitar el vaciado de la vejiga en la población adulta, particularmente en pacientes con hiperplasia benigna de próstata (50). Podrían estar indicados en pacientes con disfunción detrusor-esfínter, pero no está aprobado su uso en niños. Sin embargo, existen estudios que muestran su efectividad en esta población $(36,51)$.

Toxina botulínica: es un tratamiento de tercera línea para niños con hiperactividad idiopática del detrusor que ha sido refractaria a procedimientos no invasivos tales como uroterapia, biofeedback, neuromodulación y farmacoterapia (52). Es una neurotoxina producida por Clostridium botulinum que provoca la relajación del músculo liso de la vejiga por la inhibición de la liberación de acetilcolina en la unión presináptica (53). Se han utilizado dosis entre 5 y $12 \mathrm{l} / \mathrm{kg}(52,53)$. El efecto es temporal, de 6 a 9 meses, $\nabla$ se requieren inyecciones repetidas (54). Se ha encontrado respuesta completa en $74,2 \%$ de los hombres y $54,5 \%$ de las mujeres y respuesta parcial en $20 \%$ de los hombres y 18,2\% de las mujeres (55). Los posibles efectos adversos son retención urinaria, cistitis y riesgo de infección del tracto urinario (25).

\section{CONCLUSIÓN}

Los síntomas del tracto urinario inferior son motivos importantes de consulta en la población pediátrica, por lo que es de suma importancia familiarizarse con la nueva estandarización de la terminología establecida por la ICCS. Para hacer un buen diagnóstico se necesitan una historia clínica $y$ un examen físico completos, junto con un diario miccional, y apoyarse en ciertos estudios paraclínicos con indicaciones establecidas. Algunos niños ameritan estudios que incluyan uroflujometría, urodinamia o cistouretrografia miccional. La uroterapia es la primera línea de tratamiento y la farmacoterapia es de gran ayuda en ciertas condiciones. Existen varias opciones terapéuticas dependiendo de la sintomatología de base. Se necesitan estudios en niños para comparar los nuevos agentes farmacológicos y las distintas terapias utilizadas en LUTS. 


\section{CONFLICTOS DE INTERESES}

Ninguno por declarar.

\section{REFERENCIAS BIBLIOGRÁFICAS}

1. Vaz GT, Vasconcelos MM, Oliveira EA, Ferreira AL, Magalhães PG, Silva FM, et al. Prevalence of lower urinary tract symptoms in school-age children. Pediatr Nephrol. 2012 Apr;27(4):597-603. DOI 10.1007/ s00467-011-2028-1.

2. Akil IO, Ozmen D, Cetinkaya AC. Prevalence of urinary incontinence and lower urinary tract symptoms in school-age children. Urol J. 2014 JuI; 1 1(3):1602-8.

3. Ballek NK, McKenna PH. Lower urinary tract dysfunction in childhood. Urol Clin North Am. 2010 May;37(2):215-28. DOI 10.1016/j.ucl.2010.03.001.

4. Guerra L, Leonard $M$, Castagnetti $M$. Best practice in the assessment of bladder function in infants. Ther Adv Urol. 2014 Aug;6(4):148-64. DOI 10.1177/1756287214528745.

5. Clemens JQ. Basic bladder neurophysiology. Urol Clin North Am. 2010 Nov;37(4):487-94. DOI 10.1016/j. ucl.2010.06.006.

6. Fowler CJ, Griffiths D, de Groat WC. The neural control of micturition. Nat Rev Neurosci. 2008 Jun;9(6):45366. DOI 10.1038/nrn2401.

7. Austin PF, Bauer SB, Bower W, Chase J, Franco I, Hoebeke P, et al. The standardization of terminology of lower urinary tract function in children and adolescents: update report from the Standardization Committee of the International Children's Continence Society. J Urol. 2014 Jun;191(6):1863-65.e13. DOI 10.1016/j.juro.2014.01.110.

8. Austin PF, Bauer SB, Bower W, Chase J, Franco I, Hoebeke P, et al. The standardization of terminology of lower urinary tract function in children and adolescents: Update report from the standardization committee of the International Children's Continence Society. Neurourol Urodyn. 2016 Apr;35(4):471-81. DOI 10.1002/nau.22751.

9. Vande Walle J, Rittio S, Bauer S, Egogert P, MarschallKehrel D, Tekgul S, et al. Practical consensus guidelines for the management of enuresis. Eur J Pediatr. 2012 Jun;171(6):971-83. DOI 10.1007/s00431-012-16877. Erratum in: Eur J Pediatr. 2013 Feb;172(2):285. Eur J Pediatr. 2012 Jun;171(6):1005.
10. Hoebeke P, Bower W, Combs A, De Jong T, Yang S. Diagnostic evaluation of children with daytime incontinence. J Urol. 2010 Feb;183(2):699-703. DOI 10.1016/j.juro.2009.10.038.

11. Schäfer W, Abrams P, Liao L, Mattiasson A, Pesce F, Spangberg A, et al. Good urodynamic practices: uroflowmetry, filling cystometry, and pressure-flow studies. Neurourol Urodyn. 2002;21(3):261-74.

12. Rittio S, Kamperis K, Siggaard C, Hagstroem S, Djurhuus JC. Age related nocturnal urine volume and maximum voided volume in healthy children: reappraisal of International Children's Continence Society definitions. J Urol. 2010 Apr;183(4):1561-7. DOI 10.1016/j.juro.2009.12.046.

13. Plata Salazara M, Torres Castellanos L. El estudio urodinámico. Urol Colomb. 2014 Ago;23(2):128-38. DOI 10.1016/S0120-789X(14)50042-1.

14. Wyndaele JJ, Gammie A, Bruschini H, De Wachter S, Fry CH, Jabr RI, et al. Bladder compliance what does it represent: can we measure it, and is it clinically relevant? Neurourol Urodyn. 2011 Jun;30(5):714-22. DOI 10.1002/nau.21129.

15. McGuire EJ, Woodside JR, Borden TA, Weiss RM. Prognostic value of urodynamic testing in myelodysplastic patients. J Urol. 1981 Augo; 126(2):205-9.

16. von Gontard A. The impact of DSM-5 and guidelines for assessment and treatment of elimination disorders. Eur Child Adolesc Psychiatry. 2013 Feb;22 Suppl 1:S61-7. DOI 10.1007/s00787-012-0363-9.

17. Kamperis K, Van Herzeele C, Rittig S, Vande Walle J. Optimizing response to desmopressin in patients with monosymptomatic nocturnal enuresis. Pediatr Nephrol. 2017 Feb;32(2):217-26. DOI 10.1007/s00467016-3376-7.

18. Richardson I, Palmer LS. Successful treatment for giggle incontinence with biofeedback. J Urol. 2009 Oct;182(4 Suppl):2062-6. DOI $10.1016 / \mathrm{j}$. juro.2009.03.021

19. Yeung CK, Godley ML, Ho CK, Ransley PG, Duffy PG, Chen CN, et al. Some new insights into bladder function in infancy. Br J Urol. 1995 Aug; 76(2):235-40.

20. Nevéus T, von Gontard A, Hoebeke P, Hjälmås K, Bauer $\mathrm{S}$, Bower W, et al. The standardization of terminology of lower urinary tract function in children and adolescents: report from the Standardisation Committee of the International Children's Continence Society. J Urol. 2006 JuI; 176(1):314-24. 
21. von Gontard A, Baeyens D, Van Hoecke E, Warzak WJ, Bachmann C. Psychological and psychiatric issues in urinary and fecal incontinence. J Urol. 2011 Apr;185(4):1432-6. DOI 10.1016/j.juro.2010.11.051.

22. Combs AJ, Grafstein N, Horowitz M, Glassberg KI. Primary bladder neck dysfunction in children and adolescents I: pelvic floor electromyography lag time--a new noninvasive method to screen for and monitor therapeutic response. J Urol [Internet]. 2005 Jan [cited 2016 Sept];173(1):[207-10]; discussion 210-1. Available from: http://linkinghub.elsevier.com/retrieve/pii/ S0022534705608191

23. Baker SS, Liptak GS, Colletti RB, Croffie JM, Di Lorenzo $\mathrm{C}$, Ector $\mathrm{W}$, et al. Constipation in infants and children: evaluation and treatment. A medical position statement of the North American Society for Pediatric Gastroenterology and Nutrition. J Pediatr Gastroenterol Nutr. 1999 Nov;29(5):612-26. Erratum in: J Pediatr Gastroenterol Nutr 2000 Jan;30(1):109.

24. Chase J, Austin P, Hoebeke P, McKenna P; International Children's Continence Society. The management of dysfunctional voiding in children: a report from the Standardisation Committee of the International Children's Continence Society. J Urol. 2010 Apr;183(4):1296-302. DOI 10.1016/j.juro.2009.12.059.

25. Maternik M, Krzeminska K, Zurowska A. The management of childhood urinary incontinence. Pediatr Nephrol. 2015 Jan;30(1):41-50. DOI 10.1007/s00467014-2791-X.

26. Neveus T, Eggert P, Evans J, Macedo A, Rittig S, TekgüI S, et al. Evaluation of and Treatment for Monosymptomatic Enuresis: A Standardization Document From the International Children's Continence Society. J Urol [Internet]. 2010 Feb [cited 2016 Sept];183(2):[4417]. Available from: http://linkinghub.elsevier.com/retrieve/pii/Soo22534709026822

27. Burgers RE, Mugie SM, Chase J, Cooper CS, von Gontard A, Rittig CS, et al. Management of functional constipation in children with lower urinary tract symptoms: report from the Standardization Committee of the International Children's Continence Society. J Urol. 2013 Jul;190(1):29-36. DOI 10.1016/j. juro.2013.01.001.

28. Franco I, von Gontard A, De Gennaro M; International Childrens's Continence Society. Evaluation and treatment of nonmonosymptomatic nocturnal enuresis: a standardization document from the International Children's Continence Society. J
Pediatr Urol. 2013 Apr;9(2):234-43. DOI 10.1016/j.jpurol.2012.10.026.

29. Bauer SB, Austin PF, Rawashdeh YF, de Jong TP, Franco I, Siggard C, et al. International Children's Continence Society's recommendations for initial diagnostic evaluation and follow-up in congenital neuropathic bladder and bowel dysfunction in children. Neurourol Urodyn. 2012 Jun;31(5):610-4. DOI 10.1002/nau.22247.

30. Hagstroem S, Mahler B, Madsen B, Djurhuus JC, Rittig S. Transcutaneous electrical nerve stimulation for refractory daytime urinary urge incontinence. J Urol. 2009 Oct;182(4 Suppl):2072-8. DOI 10.1016/j. juro.2009.05.101.

31. Monga AK, Tracey MR, Subbaroyan J. A systematic review of clinical studies of electrical stimulation for treatment of lower urinary tract dysfunction. Int Urogynecol J. 2012 Aug;23(8):993-1005. DOI 10.1007/ s00192-012-1691-5.

32. Barroso U Jr, Lordêlo P. Electrical nerve stimulation for overactive bladder in children. Nat Rev Urol. 2011 Jun;8(7):402-7. DOI 10.1038/nrurol.2011.68.

33. Glazener CM, Evans JH, Peto RE. Alarm interventions for nocturnal enuresis in children. Cochrane Database Syst Rev. 2005 Apr;(2):CD002911.

34. Hegde SS, Mammen M, Jasper JR. Antimuscarinics for the treatment of overactive bladder: current options and emerging therapies. Curr Opin Investig Drugs. 2004 Jan;5(1):40-9.

35. Nijman RJ. Role of antimuscarinics in the treatment of nonneurogenic daytime urinary incontinence in children. Urology. 2004 Mar;63(3 Suppl 1):45-50.

36. Gleason JM, Daniels C, Williams K, Varghese A, Koyle MA, Bägli DJ, et al. Single center experience with oxybutynin transdermal system (patch) for management of symptoms related to non-neuropathic overactive bladder in children: an attractive, well tolerated alternative form of administration. J Pediatr Urol. 2014 Aug;10(4):753-7. DOI 10.1016/j.jpurol.2013.12.017.

37. Abrams P, Andersson KE. Muscarinic receptor antagonists for overactive bladder. BJU Int. 2007 Nov; 100(5):987-1006.

38. Jonville AP, Dutertre JP, Barbellion M, Autret E. [Adverse effects of oxybutynin chloride (Ditropan) in pediatrics]. Arch Fr Pediatr. 1993 Jan;50(1):27-9. French. 
39. Madhurrata P, Cody JD, Ellis G, Herbison GP, HaySmith EJ. Which anticholinergic drug for overactive bladder symptoms in adults. Cochrane Database Syst Rev. 2012 Jan;1:CD005429. DOI 10.1002/14651858. CD005429.pub2.

40. Bolduc S, Upadhyay J, Payton J, Bägli DJ, McLorie GA, Khoury $\mathrm{AE}$, et al. The use of tolterodine in children after oxybutynin failure. BJU Int. 2003 Mar;91(4):398-401.

41. HjäImås K, Hellström AL, Mogren K, Läckogren G, Stenberg A. The overactive bladder in children: a potential future indication for tolterodine. BJU Int. 2001 Apr;87(6):569-74.

42. Hoebeke P, De Pooter J, De Caestecker K, Raes A, Dehoorne J, Van Laecke E, et al. Solifenacin for therapy resistant overactive bladder. J Urol. 2009 Oct;182(4 Suppl):2040-4. DOI 10.1016/j.juro.2009.05.100.

43. Bolduc S, Moore K, Nadeau G, Lebel S, Lamontagne P. Hamel M. Prospective open label study of solifenacin for overactive bladder in children. J Urol. 2010 Oct;184(4 Suppl):1668-73. DOI 10.1016/j. juro.2010.03.124.

44. Van de Walle J, Van Herzeele C, Raes A. Is there still a role for desmopressin in children with primary monosymptomatic nocturnal enuresis?: a focus on safety issues. Drug Saf. 2010 Apr;33(4):261-71. DOI 10.2165/11319110-000000000-00000.

45. De Guchtenaere A, Van Herzeele C, Raes A, Dehoorne J, Hoebeke P, Van Laecke E, et al. Oral lyophylizate formulation of desmopressin: superior pharmacodynamics compared to tablet due to low food interaction. J Urol. 2011 Jun;185(6):2308-13. DOI 10.1016/j. juro.2011.02.039.

46. Marschall-Kehrel D, Harms TW; Enuresis Algorithm of Marschall Survey Group. Structured desmopressin withdrawal improves response and treatment outcome for monosymptomatic enuretic children. J Urol. 2009 Oct;182(4 Suppl):2022-6. DOI 10.1016/j. juro.2009.03.068.
47. Ohtomo Y, Umino D, Takada M, Fujinaga S, Niijima $S$, Shimizu T. Gradual tapering of desmopressin leads to better outcome in nocturnal enuresis. Pediatr Int. 2015 Aug;57(4):656-8. DOI 10.1111/ped.12614.

48. Lucchini B, Simonetti GD, Ceschi A, Lava SA, Faré PB, Bianchetti MG. Severe signs of hyponatremia secondary to desmopressin treatment for enuresis: a systematic review. J Pediatr Urol. 2013 Dec;9(6 Pt B):104953. DOI 10.1016/j.jpurol.2013.02.012.

49. Hunsballe JM, Rittio S, Pedersen EB, Olesen OV, Djurhuus JC. Single dose imipramine reduces nocturnal urine output in patients with nocturnal enuresis and nocturnal polyuria. J Urol. 1997 Sep;158(3 Pt 1):830-6.

50. Mombini H. The relationship between weight as well as the kind of prostate hypertrophy and the response to Tamsulosine, a specific alpha-blocker. Urol J. 2004 Spring; 1(2):115-6.

51. Donohoe JM, Combs AJ, Glassberg KI. Primary bladder neck dysfunction in children and adolescents II: results of treatment with alpha-adrenergic antagonists. J Urol [Internet]. 2005 Jan[cited 2016 Sept];173(1):[212-6]. Available from: http://linkinghub. elsevier.com/retrieve/pii/S0022534705608208

52. DasGupta R, Murphy FL. Botulinum toxin in paediatric urology: a systematic literature review. Pediatr Surg Int. 2009 Jan;25(1):19-23. DOI 10.1007/s00383-008-2260-3.

53. Dmochowski R, Sand PK. Botulinum toxin A in the overactive bladder: current status and future directions. BJU Int. 2007 Feb;99(2):247-62.

54. Hoebeke P, De Caestecker K, Vande Walle J, Dehoorne J, Raes A, Verleyen P, et al. The effect of botulinum-A toxin in incontinent children with therapy resistant overactive detrusor. J Urol. 2006 Jul; 176(1):328-30; discussion 330-1.

55. McDowell DT, Noone D, Tareen F, Waldron M, Quinn F. Urinary incontinence in children: botulinum toxin is a safe and effective treatment option. Pediatr Surg Int. 2012 Mar;28(3):315-20. DOI 10.1007/s00383-011-3039-5. 